\title{
Comparison of intrarenal pelvic pressure and postoperative fever between standard- and mini-tract percutaneous nephrolithotomy: a systematic review and meta-analysis of randomized controlled trials
}

\author{
Dechao Feng", Xiongfeng Zeng", Ping Han, Xin Wei \\ Department of Urology, Institute of Urology, West China Hospital, Sichuan University, Chengdu 610041, China \\ Contributions: (I) Conception and design: D Feng; (II) Administrative support: P Han; (III) Provision of study materials or patients: D Feng, X Zeng; \\ (IV) Collection and assembly of data: D Feng, X Zeng; (V) Data analysis and interpretation: D Feng, X Zeng; (VI) Manuscript writing: All authors; \\ (VII) Final approval of manuscript: All authors. \\ \#These authors contributed equally to this work. \\ Correspondence to: Xin Wei, PhD. Department of Urology, Institute of Urology, West China Hospital, Sichuan University, Guoxue Xiang \#37, \\ Chengdu 610041, China. Email: xweiwch@126.com.
}

Background: High intrarenal pelvic pressure (IPP) induces systemic absorption of irrigation fluid containing bacteria or endotoxins, which is associated with postoperative fever (POF) and even urosepsis. The emphasis of this meta-analysis lies in comparison of IPP and POF between mini-tract percutaneous nephrolithotomy (MPCNL) and standard-tract percutaneous nephrolithotomy (SPCNL).

Methods: Eligible randomized controlled trials (RCTs) were identified from electronic databases from inception to November 2019. Studies selection, quality assessment, data extraction and analysis were accomplished by two independent reviewers using Cochrane Collaboration's tools.

Results: Patients in the MPCNL group experienced higher IPP compared to SPCNL group [mean difference (MD): 8.31, 95\% confidence interval (CI): 3.67-12.96, $\mathrm{P}=0.0005]$ with highly between-study heterogeneity $\left(\mathrm{P}=0.001, \mathrm{I}^{2}=85 \%\right)$. Notably, the IPP was higher in MPCNL group in different stages of the procedure including introduction, fragmentation and end. However, only two trials were available for pooled analysis. Additionally, the risk of POF in MPCNL was 2.43 times higher than that in SPCNL [odds ratio (OR): 2.43 , 95\% CI: 1.39-4.27, $\mathrm{P}=0.002]$ with no significant between-study heterogeneity $\left(\mathrm{P}=0.83, \mathrm{I}^{2}=0 \%\right)$. The two procedures was comparable on stone-free rate (SFR) (OR: 1.06, 95\% CI: 0.61-1.86, $\mathrm{P}=0.83$ ) and operation time (MD: $5.69,95 \% \mathrm{CI}:-4.54$ to $15.91, \mathrm{P}=0.28$ ).

Conclusions: Current evidence indicates that MPCNL is an effective alternative to SPCNL with comparable SFR. IPP and POF is significantly higher during MPCNL compared to SPCNL. Intraoperative detection of IPP is of great significance for ensuring safety and reducing postoperative complications, especially for patients with MPCNL and the duration of stone fragmentation. Further large well-designed trials are warranted to confirm our findings.

Keywords: Intrarenal pelvic pressure (IPP); postoperative fever (POF); percutaneous nephrolithotomy (PCNL); meta-analysis

Submitted Dec 08, 2019. Accepted for publication Mar 06, 2020.

doi: $10.21037 /$ tau.2020.03.30

View this article at: http://dx.doi.org/10.21037/tau.2020.03.30 


\section{Introduction}

Currently, percutaneous nephrolithotomy (PCNL) has been an established procedure primarily used to treat urinary calculi greater than $2 \mathrm{~cm}$ due its higher stone clearance and cost-effectiveness when compared to other alternative procedures such as extracorporeal shock wave lithotripsy (ESWL) and retrograde intrarenal surgery (RIRS) (1). Recently, modifications of standard PCNL (SPCNL) technique, namely minimally invasive PCNL (MPCNL), have been proposed by some experts as alternatives to decrease the operation-related complications through smaller renal access sheaths, such as micro-PCNL (4.5 Fr outer sheath), ultra-MPCNL (7.5 Fr nephroscope and 11-13 Fr outer sheath), and super-MPCNL (7.5 Fr nephroscope and modified 10-14 Fr outer sheath) (2). These improved techniques are also known as "mini perc" or "mini-PCNL".

Our latest meta-analysis (3) comparing MPCNL to SPCNL showed that MPCNL has the advantage of less bleeding, fewer transfusion and higher stone-free rate (SFR) but the disadvantage of a longer time to dislodge stones. Besides, there was no significant difference between the two groups for renal stones $\geq 2 \mathrm{~cm}$ in terms of SFR. However, we did not assess the effect of intrarenal pelvic pressure (IPP) on surgical safety. It is common for urologists to pressurize the irrigation to provide a clear field of view and facilitate removal of the calculi fragments (4). Unfortunately, poor drainage of irrigation may provisionally increase IPP, and this may cause systemic absorption of bacteria and endotoxins from the irrigation fluid, further triggering postoperative fever (POF) and sepsis $(4,5)$. In this situation, a systematic review and meta-analysis of existing controlled studies was conducted to explore this relationship between MPCNL and SPCNL.

\section{Methods}

\section{Study selection}

Eligible randomized controlled trials (RCTs) were identified from electronic databases including PubMed, the Cochrane Library, Embase, Web of Science and WANFANG up to November 2019 in accordance with the PRISMA guidelines (6) with no limitation to the language. Additionally, the manual searches of the reference lists of all related articles were supplemented to broaden the search. The following keywords or Mesh terms were searched in title/abstract: "pelvic pressure" and "percutaneous nephrolithotomy". All trials reporting the outcomes of interest were included. A statement of ethics approval and patient inform consent was not required because all studies included in this article was derived from published records online.

\section{Selection criteria}

The eligibility of studies was defined using the PICOS method: Patients (P): kidney stone; Intervention (I): PCNL; Comparison (C): publications comparing MPCNL to SPCNL; Outcomes (O): the primary outcomes were IPP, POF, procalcitonin and white blood cell; the secondary outcomes were SFR, operation time and bleeding; Study design (S): RCTs. The present metaanalysis only reported IPP, POF, SFR and operation time, because only a few studies described bleeding, procalcitonin and white blood cell so that we are unable to conduct a meta-analysis.

\section{Quality assessment}

Two independent reviewers (D Feng, X Zeng) evaluated the study quality (Figure 1) according to the Cochrane Collaboration's Risk of Bias tool in Review Manager software (https://community.cochrane.org/help/toolsand-software/revman-5). This tool primarily evaluates 7 domains: random sequence generation (selection bias); allocation concealment (selection bias); blinding of participants and personnel (performance bias); blinding of outcome assessment (detection bias); incomplete outcome data (attrition bias); selective reporting (reporting bias); other bias (such as funding sources). Two independent investigators (D Feng, X Zeng) screened study based on titles and abstracts. Studies that satisfied the inclusion criteria were retrieved for full-text assessment. Data were independently extracted by two reviewers (D Feng, X Zeng). Disagreements were resolved by another researchers (P Han). The manuscript was revised by the reviewer (X Wei).

\section{Statistical analysis}

The continuous and dichotomous variables were described as means \pm standard deviation $(\mathrm{SD})$ and proportions, respectively. Median and range were used to estimate mean and SD (7). The percentiles, 25 th and 75 th percentiles as well as 5 th and 95 th percentiles, were transformed to SD through the following formula: $\mathrm{SD} \approx$ Norm IQR $=(\mathrm{P} 75-\mathrm{P} 25) \times 0.7413$ (IQR: interquartile range, $\mathrm{P} 75$ : 75 th percentile, $\mathrm{P} 25: 25$ th percentile) (8). 


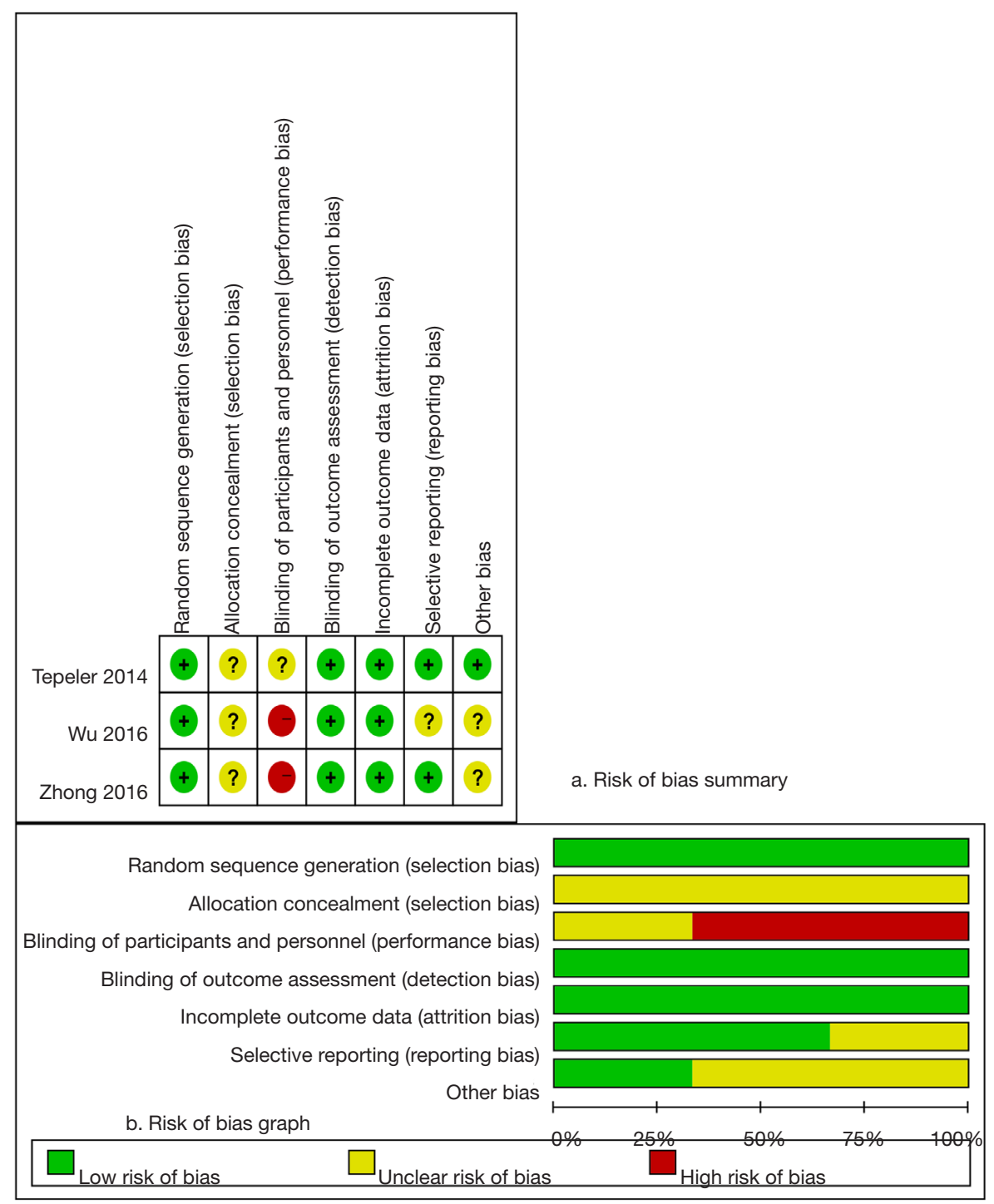

Figure 1 The risk of bias using Cochrane Collaboration's tools.

We calculated pooled estimates of the mean difference (MD) or standard mean differences (SMD) and odds ratio (OR) for continuous and dichotomous variables, respectively. We used the Cochran Q test to evaluate between-study heterogeneity (9). We also did $\mathrm{I}^{2}$ testing to assess the magnitude of the heterogeneity with values $\leq 50 \%$ regarded as being acceptable heterogeneity (10). The fixed effects model was used unless there exists heterogeneity $(\mathrm{P}<0.1)$, and significance was set at $\mathrm{P}<0.05$. This meta-analysis was accomplished by RevMan5 (version 5.3).

\section{Results}

\section{Search results}

We identified 95 possible studies and three trials (11-13) reporting the outcomes of interest were included in the final analysis after duplicates removed, titles and abstracts screened and full-text articles assessed. The three trials (11-13) contained 331 patients (167 in the MPCNL and 164 in the SPCNL), and the number of males was 199. The study selection process is shown in Figure 2, and Table 1 details 


\section{PRISMA 2009 Flow Diagram (14)}

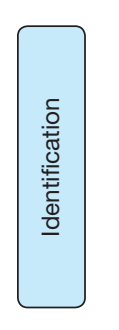

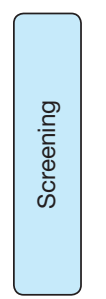

Records identified through database searching English [85]; Chinese [8]
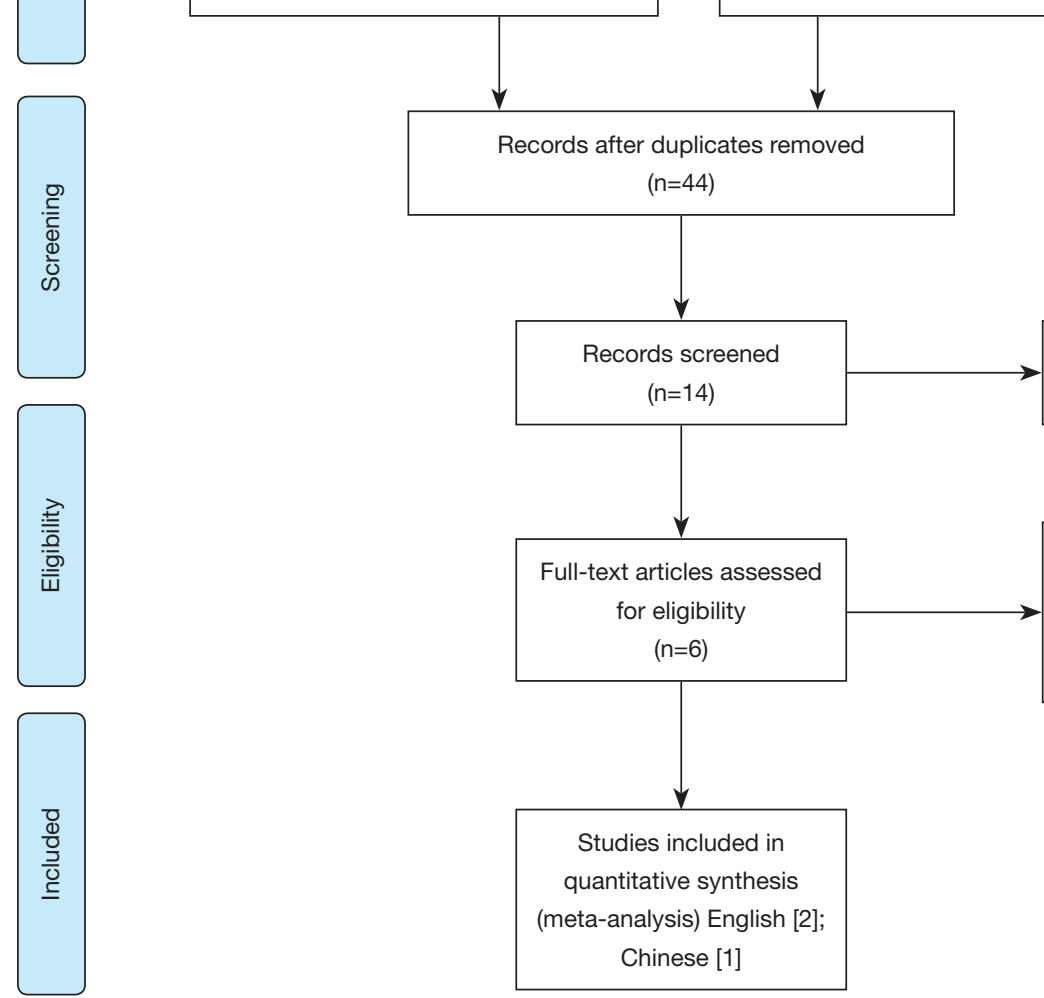

Additional records identified through other sources $(n=2)$

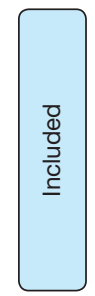

Full-text articles excluded, with reasons $(n=3)$ : (I) Meta-analysis; (II) Non-human trials;

Figure 2 The study selection process (14).

the main characteristics of included studies.

\section{The primary outcomes}

Patients in the MPCNL group experienced higher IPP compared to SPCNL group (MD: 8.31, 95\% CI: $3.67-$ $12.96, \mathrm{P}=0.0005)$ with highly between-study heterogeneity $\left(\mathrm{P}=0.001, \mathrm{I}^{2}=85 \%\right)$. The trial conducted by $\mathrm{Wu}$ et al. (11) was source of heterogeneity. This may be resulted from different irrigation flow and IPP measurement. Notably, the IPP was higher in MPCNL group in different stages of the procedure including introduction (MD: 2.84, 95\% CI: 2.33-3.34, $\mathrm{P}<0.00001$ ), fragmentation (MD: 9.59, 95\% CI: 4.40-14.77, $\mathrm{P}=0.0003)$ and end (MD: $5.72,95 \% \mathrm{CI}:-0.75$ to $12.18, \mathrm{P}=0.08)$. However, only two trials $(12,13)$ were available for pooled analysis. Additionally, the risk of POF in MPCNL was 2.43 times higher than that in SPCNL (OR:2.43, 95\% CI: 1.39-4.27, $\mathrm{P}=0.002$ ) with no significant between-study heterogeneity $\left(\mathrm{P}=0.83, \mathrm{I}^{2}=0 \%\right)$.

\section{The secondary outcomes}

The efficacy of the two procedures on SFR was comparable (OR: 1.06, 95\% CI: 0.61-1.86, $\mathrm{P}=0.83$ ). Besides, pooling data of three studies (11-13) that assessed operation time in 331 patients showed there is no statistically significant difference in the MPCNL versus SPCNL group (MD: 5.69, 95\% CI: -4.54 to $15.91, \mathrm{P}=0.28)$ with significant between- 


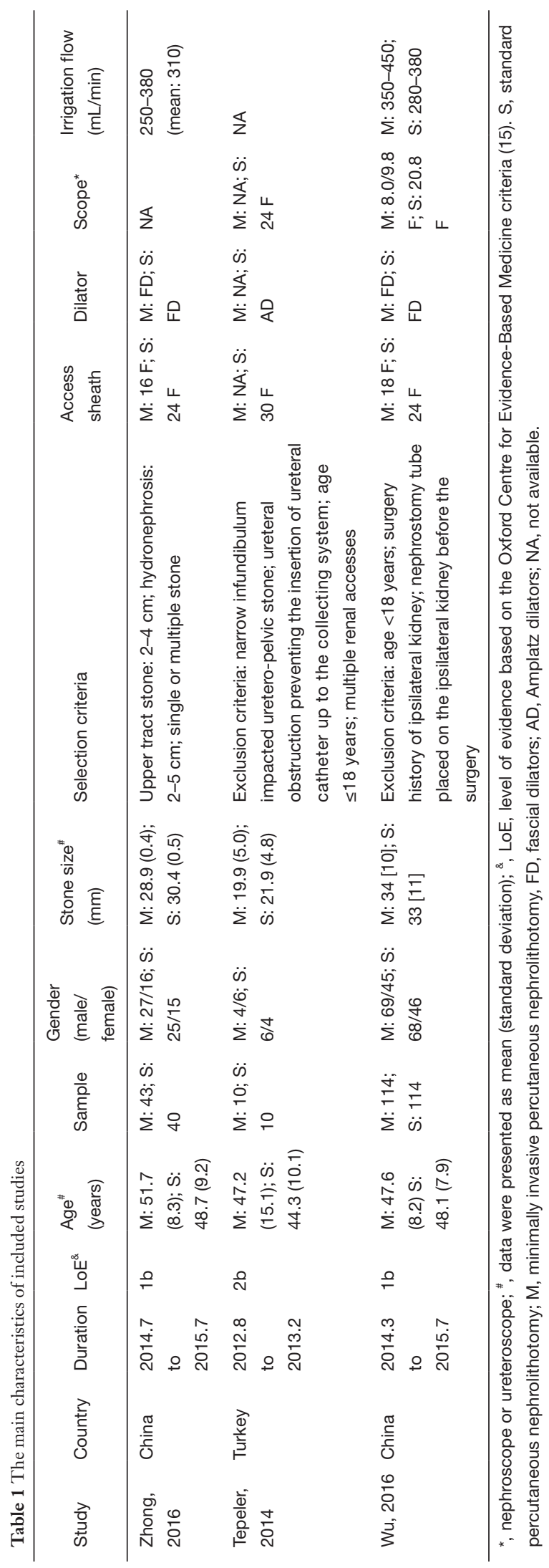

study heterogeneity. The pooled results of the primary and secondary outcomes are summarized in Figure 3.

\section{Discussion}

Kidney stone is a common urinary disease, and the goal of treatment is to remove the stone as much as possible and protect the renal function. At the present, PCNL has been the most extensively used procedure for the treatment of renal stone larger than $2 \mathrm{~cm}$ (1). The first PCNL technique was described by Fernstrom and Johansson in 1976 (16). This procedure removed calculus from the renal collecting system through percutaneous access tracts with a nephoscopy or ureteroscope $(4,16)$. Owing to the large trauma to renal parenchyma and severe complications like bleeding, Jackman et al. (17) firstly introduced the "miniperc" technique in children in 1998 and Lahme and colleagues (18) shared their experience of mini-PCNL with a 12 -French rigid nephroscope and 15 -French Amplatz sheath firstly in adults in 2001. Subsequently, the improvements continued over the past decades with the introduction of micro-PCNL (19), ultra-MPCNL (20) and super MPCNL (21) techniques. Technically speaking, MPCNL has a smaller percutaneous tract and a smaller field of vision compared to SPCNL. Additionally, MPCNL requires special equipment and is more difficult to operate. However, this was not the case. MPCNL sacrifices its technical advantages to achieve higher surgical results. Specifically, MPCNL has advantage of decreased trauma to renal parenchyma, less pain, less bleeding and shorter length of stay than SPCNL which has been demonstrated by the previous meta-analysis (3). Elevated IPP might be attributed to incomplete positioning of the nephroscopy sheath within the collecting system and endoscopy through a narrow infundibulum (22). Moreover, Zhong and his colleagues (4) found that smaller tract contributes to higher IPP and POF rate by comparing IPP of different percutaneous tracts (14- to 18-French). The possible mechanisms of increased IPP induced by MPCNL may be attributable to the following aspects. Firstly, irrigation outflow principally goes through the interspace between scope and peel-away sheath, and little was passed through the ureter (4). Consequently, smaller tracts are prone to cause poor drainage of irrigation outflow from the interspace between endoscope and peel-away sheath. Secondly, we use the impulsive stream with high pressure produced by microcomputer control pump to prompt removal of the calculi fragments, and we continuously pressurize the irrigation to provide a clear 


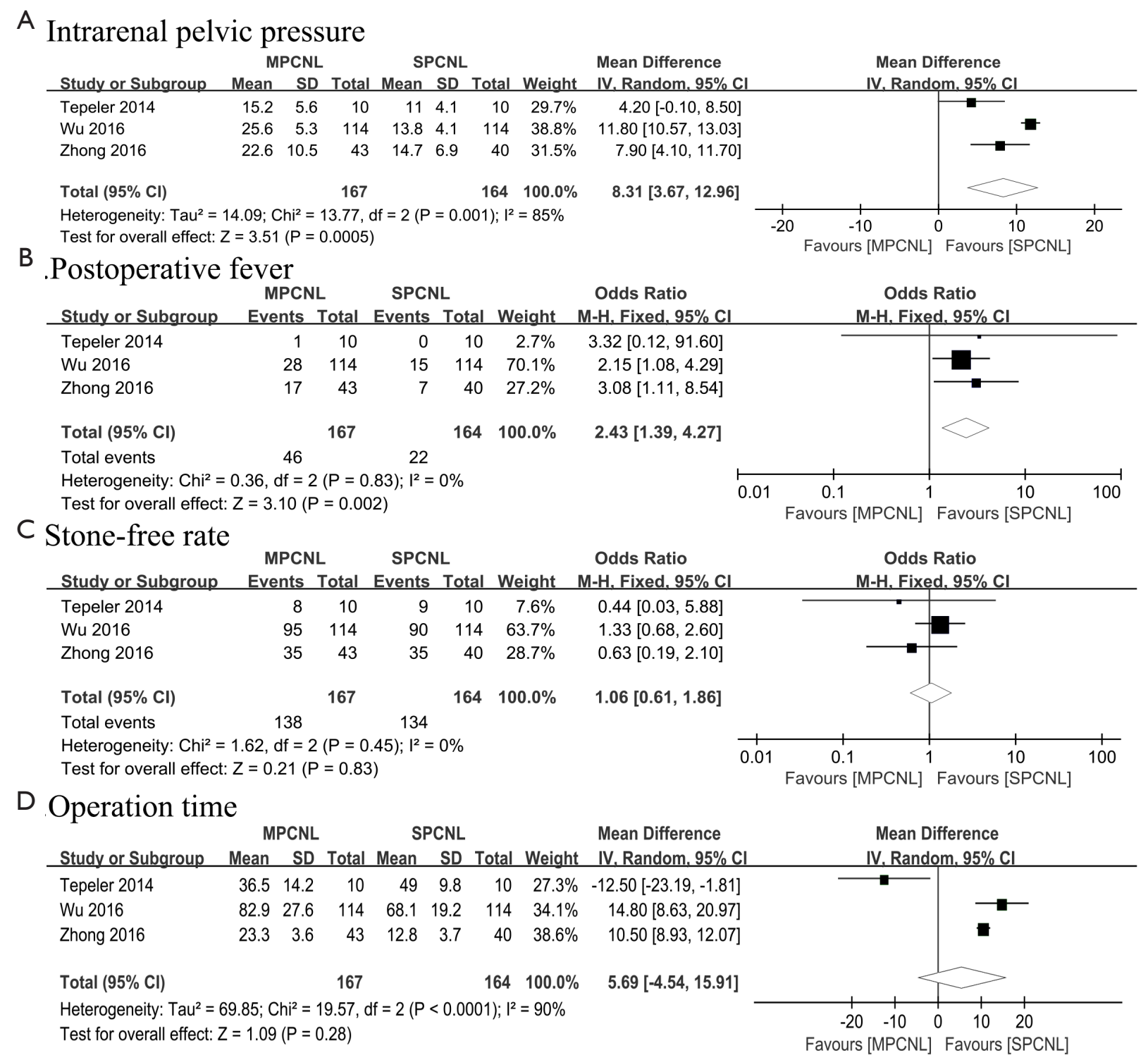

Figure 3 The pooled outcomes included in this meta-analysis: (A) intrarenal pelvic pressure; (B) postoperative fever; (C) stone-free rate; (D) operation time. MPCNL, minimally invasive percutaneous nephrolithotomy; SPCNL, standard-tract percutaneous nephrolithotomy; $\mathrm{SD}$, standard deviation; CI, confidence interval.

field of view for the urologists throughout the period of MPCNL (4). Besides, the complexity of operation may also contribute to the elevation of IPP. High renal pelvic pressure results in systemic absorption of irrigation fluid containing bacteria or endotoxins, which induces POF $(4,5)$. Although the previous meta-analysis (3) indicated that MPCNL was a safe and effective alternative to SPCNL, IPP should not be ignored during PCNL. Currently, little was known about IPP and its association with POF between MPCNL and SPCNL. Thus, the emphasis of this meta-analysis lies in comparison of IPP and POF between MPCNL and SPCNL.

Our study showed that MPCNL has higher IPP than SPCNL throughout the period of operation which is consistent with the result of POF. Actually, the pressure in the renal pelvis is about $7.35 \mathrm{mmHg}$ under physiological condition, and the IPP larger than $30 \mathrm{mmHg}$ will lead to continuous liquid reflux in the renal pelvis (23). In order 
to reduce the backflow of perfusion fluid, $30 \mathrm{mmHg}$ is the maximal safe value during PCNL (22). The regurgitation pathway mainly includes renal pelvic tubular regurgitation and renal calyceal fornix regurgitation, the latter includes renal pelvic and renal sinus regurgitation, renal perivascular regurgitation, renal pelvic venous regurgitation, renal pelvic lymphatic regurgitation and so on (22).

Similar to the previous meta-analysis (3), our study showed no significant difference in SFR between MPCNL and SPCNL in terms of renal stones $\geq 2 \mathrm{~cm}$. Therefore, our study confirms the efficacy of MPCNL. Notably, IPP generally remains lower than the backflow level $(30 \mathrm{mmHg})$ during $\mathrm{MPCNL}$ via a 14 - to 18 -French percutaneous tract (4). Any factors that caused poor drainage would result in temporarily elevated IPP greater than $30 \mathrm{mmHg}$, and many such occurrences of high pressure would have an accumulating effect, which means enough backflow to cause bacteremia and POF (4). Specifically, the threshold of accumulated time of IPP greater than $30 \mathrm{mmHg}$ may be $50 \mathrm{~s}$ (4). Besides, there was also no significant difference in operation time between the two groups, which was different from the previous study (3). However, due to the limited RCTs and great heterogeneity, we are unable to make definitive conclusions.

To our knowledge, our study is the first meta-analysis that compares IPP and POF between MPCNL and SPCNL. However, our present study does have the following limitations. Foremost, the broad heterogeneity in study populations, designs and definitions of outcome measures. Secondly, we are unable to perform a meta-analysis on hematology-related infection indicators such as procalcitonin and white blood cell due to limited RCTs and unavailable data, despite these indicators seemed to be higher in MPCNL group $(11,13)$. Thirdly, the limited number of RCTs and sample size make our conclusions unstable and unreliable.

Although MPCNL has been established to be an alternative to SPCNL, it is unlikely to completely replace SPCNL due to the limited sheath diameter. MPCNL represents an extension of the indication for SPCNL that it can replace in no way, and smaller tracts do not mean better effects. Too small tract might not provide enough interspace between the scope and pee-away sheath for irrigation outflow, and thereby increase IPP causing POF and even sepsis. We should pay attention to the cumulative effect of IPP during MPCNL. Besides, the relationship between percutaneous tract and the number, size, type and components of stone remains the focus of research in the future.

\section{Conclusions}

Current evidence indicates that MPCNL is an effective alternative to SPCNL with comparable SFR. IPP and POF is significantly higher during MPCNL compared to SPCNL. Intraoperative detection of IPP is of great significance for ensuring safety and reducing postoperative complications, especially for patients with MPCNL and the duration of stone fragmentation. Further large welldesigned trials are warranted to confirm our findings.

\section{Acknowledgments}

Funding: The study was supported by the 1.3 .5 project for disciplines of excellence, West China Hospital, Sichuan University (ZY2016104) and Pillar Program from Department of Science and Technology of Sichuan Province (2018SZ0219).

\section{Footnote}

Conflicts of Interest: All authors have completed the ICMJE uniform disclosure form (available at http://dx.doi. org/10.21037/tau.2020.03.30). The authors have no conflicts of interest to declare.

Etbical Statement: The authors are accountable for all aspects of the work in ensuring that questions related to the accuracy or integrity of any part of the work are appropriately investigated and resolved.

Open Access Statement: This is an Open Access article distributed in accordance with the Creative Commons Attribution-NonCommercial-NoDerivs 4.0 International License (CC BY-NC-ND 4.0), which permits the noncommercial replication and distribution of the article with the strict proviso that no changes or edits are made and the original work is properly cited (including links to both the formal publication through the relevant DOI and the license). See: https://creativecommons.org/licenses/by-nc-nd/4.0/.

\section{References}

1. Türk C, Skolarikos A, Neisius A, et al. Guidelines on urolithiasis. The Netherlands: European Association of Urology. Access date (2019). Available online: https:// uroweb.org/guideline/urolithiasis/\#note_259. 
2. Güler A, Erbin A, Ucpinar B, et al. Comparison of miniaturized percutaneous nephrolithotomy and standard percutaneous nephrolithotomy for the treatment of large kidney stones: a randomized prospective study. Urolithiasis 2019;47:289-95.

3. Feng D, Hu X, Tang Y, et al. The efficacy and safety of miniaturized percutaneous nephrolithotomy versus standard percutaneous nephrolithotomy: A systematic review and meta-analysis of randomized controlled trials. Investig Clin Urol 2020;61:115-26.

4. Zhong $\mathrm{W}$, Zeng $\mathrm{G}, \mathrm{Wu} \mathrm{K}$, et al. Does a smaller tract in percutaneous nephrolithotomy contribute to high renal pelvic pressure and postoperative fever? J Endourol 2008;22:2147-51.

5. Kreydin EI, Eisner BH. Risk factors for sepsis after percutaneous renal stone surgery. Nat Rev Urol 2013;10:598-605.

6. Moher D, Shamseer L, Clarke M, et al. Preferred reporting items for systematic review and meta-analysis protocols (PRISMA-P) 2015 statement. Syst Rev 2015;4:1.

7. Hozo SP, Djulbegovic B, Hozo I. Estimating the mean and variance from the median, range, and the size of a sample. BMC Med Res Methodol 2005;5:13.

8. Yang L, Wang G, Du Y, et al. Remote ischemic preconditioning reduces cardiac troponin I release in cardiac surgery: a meta-analysis. J Cardiothorac Vasc Anesth 2014;28:682-9.

9. Higgins JP, Thompson SG, Deeks JJ, et al. Measuring inconsistency in meta-analyses. BMJ 2003;327:557-60.

10. Higgins JP, Thompson SG. Quantifying heterogeneity in a meta-analysis. Stat Med 2002;21:1539-58.

11. Wu C, Hua LX, Zhang JZ, et al. Comparison of renal pelvic pressure and postoperative fever incidence between standard- and mini-tract percutaneous nephrolithotomy. Kaohsiung J Med Sci 2017;33:36-43.

12. Tepeler A, Akman T, Silay MS, et al. Comparison of intrarenal pelvic pressure during micro-percutaneous nephrolithotomy and conventional percutaneous

Cite this article as: Feng D, Zeng X, Han P, Wei X. Comparison of intrarenal pelvic pressure and postoperative fever between standard- and mini-tract percutaneous nephrolithotomy: a systematic review and meta-analysis of randomized controlled trials. Transl Androl Urol 2020;9(3):11591166. doi: $10.21037 /$ tau.2020.03.30 nephrolithotomy. Urolithiasis 2014;42:275-9.

13. Zhong ZG, Pan TJ, Li GC. Comparison of intrarenal pelvic pressure during F24 channel and F16 channel for percutaneous nephrolithotomy. Chinese Journal of Urology 2016;37:354-7.

14. Moher D, Liberati A, Tetzlaff J, et al. Preferred reporting items for systematic reviews and meta-analyses: the PRISMA statement. PLoS Med 2009;6:e1000097.

15. Phillips B, Ball C, Sacket D, et al. Oxford Centre for Evidence-based Medicine Levels of Evidence. Access date: May 1-2, 2009. Available online: https://www.mendeley. com/catalogue/oxford-centre-evidencebased-medicinelevels-evidence/

16. Fernström I, Johansson B. Percutaneous pyelolithotomy. A new extraction technique. Scand J Urol Nephrol 1976;10:257-9.

17. Jackman SV, Docimo SG, Cadeddu JA, et al. The "miniperc" technique: a less invasive alternative to percutaneous nephrolithotomy. World J Urol 1998;16:371-4.

18. Lahme S, Bichler KH, Strohmaier WL, et al. Minimally invasive PCNL in patients with renal pelvic and calyceal stones. Eur Urol 2001;40:619-24.

19. Desai MR, Sharma R, Mishra S, et al. Single-step percutaneous nephrolithotomy (microperc): the initial clinical report. J Urol 2011;186:140-5.

20. Desai J, Solanki R. Ultra-mini percutaneous nephrolithotomy (UMP): one more armamentarium. BJU Int 2013;112:1046-9.

21. Zeng G, Wan S, Zhao Z, et al. Super-mini percutaneous nephrolithotomy (SMP): a new concept in technique and instrumentation. BJU Int 2016;117:655-61.

22. Troxel SA, Low RK. Renal intrapelvic pressure during percutaneous nephrolithotomy and its correlation with the development of postoperative fever. J Urol 2002;168:1348-51.

23. Holst U, Dissing T, Rawashdeh YF, et al. Norepinephrine inhibits the pelvic pressure increase in response to flow perfusion. J Urol 2003;170:268-71. 